\title{
INFLUÊNCIA DA IDADE NA GERAÇÃO DE MODELOS DE ESPECTROSCOPIA NIR, PARA PREDIÇÃO DE PROPRIEDADES DA MADEIRA DE Eucalyptus spp. ${ }^{1}$
}

\begin{abstract}
Flaviana Reis Milagres², José Lívio Gomide ${ }^{3}$, Andreia Magaton ${ }^{4}$ e Humberto Fantuzzi Neto ${ }^{5}$
RESUMO - O objetivo deste trabalho foi o desenvolvimento de modelos NIRS para predizer as propriedades físicas e químicas de madeiras de Eucalyptus, com idades variando de 3 a 7 anos. Foram utilizadas 136 amostras e realizadas análises laboratoriais de densidade básica, teores de lignina e extrativos. Amostras de cavacos foram moídas em moinho tipo ciclone, para coleta de espectros NIR. Os modelos foram desenvolvidos por regressão de mínimos quadrados parciais (PLS), sendo testadas transformações matemáticas. Para validação dos modelos, foram utilizados grupos de amostras externas com diferentes idades, que não participaram no desenvolvimento do modelo. Os modelos desenvolvidos resultaram em consideráveis coeficientes de determinação para densidade básica $\left(R^{2} 0,84\right.$ a 0,89$)$ e lignina $\left(R^{2} 0,72\right.$ a 0,88$)$, mas os melhores valores foram encontrados para o teor de extrativos $\left(R^{2} 0,92\right.$ a 0,98$)$. Pelos valores de relação de desempenho de desvio (RPD), verificou-se que amostras com idades diferentes das utilizadas no desenvolvimento dos modelos não apresentaram boas predições, exceto para densidade básica cujos modelos desenvolvidos especificamente para as idades de 3 e 7 anos, predisseram satisfatoriamente amostras com idades entre 4 e 6 anos.
\end{abstract}

Palavras-chave: Espectroscopia de infravermelho próximo; Densidade básica; Idade.

\section{THE INFLUENCE OF THE AGE ON NEAR INFRARED SPECTROSCOPY MODELS TO PREDICTION OF THE EUcalyptus spp. WOOD PROPERTIES}

\begin{abstract}
The objective of this work was the development of NIRS models for physical and chemical properties of Eucalyptus wood at the ages 3-7 years. A total of 136 samples were collected for laboratory analyzes of basic density, lignin and extractives. Chip samples were ground in a cyclone mill to collect NIR spectra. The models were developed by partial least squares (PLS) regression and tested mathematical transformations. For model validation were used external samples of different ages. The developed models resulted in significant coefficients of determination $\left(R^{2}\right)$ for basic density (from 0.84 to 0.89 ) and lignin (from 0.72 to 0.88 ), but the best $R^{2}$ values were found for the extractives content (from 0, 92-.98). By the values of ratio of performance to deviation (RPD) were assessed that samples of different ages used in development of the models did not show good predictions. But the basic density showed, for models specifically designed for the ages of 3 and 7 years, satisfactory predictions using samples at the ages 46 years.
\end{abstract}

Keywords: Near infrared spectroscopy; Basic density; Age.

\footnotetext{
${ }^{1}$ Recebido em 11.12.2012 aceito para publicação em 06.11.2013.

${ }^{2}$ Universidade Federal de Viçosa, Centro de Ciências Agrárias, Departamento de Engenharia Florestal. E-mail: <flavianamilagres@gmail.com>.

${ }^{3}$ Universidade Federal de Viçosa, Centro de Ciências Agrárias, Departamento de Engenharia Florestal. E-mail: $<$ jlgomide@ufv.br>.

${ }^{4}$ Universidade Federal do Recôncavo da Bahia, Centro de Ciências Exatas e Tecnológicas. E-mail:<anmagaton@yahoo.com.br> .

${ }^{5}$ Universidade Federal do Espírito Santo. E-mail:<hfantuzzi@yahoo.com.br>.
} 


\section{INTRODUÇÃO}

O Brasil é hoje uma das grandes potências na produção de celulose e papel, destacando-se como o maior produtor de fibra curta branqueada do mundo. Atualmente, na produção mundial de celulose o Brasil ocupa o quarto lugar, com 13,9 milhões de toneladas produzidas, e passou do $11^{\circ}$ lugar para o $9^{\circ}$ maior produtor de papel do planeta, com 10,2 milhões de toneladas (BRACELPA, 2013). A manutenção e crescimento da competitividade do setor de celulose e papel brasileiro se baseiam na biomassa de Eucalyptus, que depende de um trabalho estratégico entre os setores privados, científicos e governamentais.

Os programas de melhoramento florestal utilizam da alta variabilidade do Eucalyptus, principal matériaprima para produção de celulose, como fonte constante para seleção de árvores com qualidades de madeira superiores à média da população. No entanto, para avaliação da qualidade da madeira é necessário que esta esteja em idade apropriada de corte, para o que leva de cinco a sete anos. A necessidade de tempo para incorporação de características de grande interesse na produção de celulose obriga os programas de melhoramento florestal a procurarem soluções para diminuir o tempo de espera na seleção de amostras e também os custos para analisar milhares de árvores. A alternativa seria a redução da idade da madeira destinada à avaliação de características tecnológicas, ou seja, a seleção precoce, que representa solução na economia de tempo para seleção de características da madeira (SILVAJÚNIOR; BRAGA, 1997).Alternativa complementar seria o uso de técnicas de baixo custo e rapidez para avaliar essas características, como a espectroscopia de infravermelho próximo (Near Infrared Spectroscopy - NIRS). A técnica NIRS, nos últimos 15 a 25 anos, tem despertado crescente interesse nas pesquisas, provando ser ferramenta útil nos estudos para agricultura, indústria alimentícia, farmacêutica, química, polímeros e petrolífera. Segundo Bokobza (1998), o “despertar deste gigante" tem surgido de melhorias na instrumentação e análises de dados. Entre as vantagens oferecidas pelo NIRS, a velocidade, simplicidade de preparação das amostras e baixo custo da técnica podem ser mencionados.

A técnica NIRS tem provado ser ferramenta útil nos programas de melhoramento para seleção de espécies envolvendo grande número de árvores (SCHIMLECK et al., 2004), e os pesquisadores pioneiros dessa técnica em madeira foram Birkett e Gambino (1998), Easty et al. (1990) e Wright et al. (1990). Muitos trabalhos relacionam a utilização de NIRS para análises de propriedades químicas da madeira como lignina e extrativos (BRINKMANN et al., 2002; GIERLINGER et al., 2002; BOERIU et al., 2004; ALVES et al., 2006) e de propriedades físicas como a densidade básica (HOFFMEYER; PEDERSEN, 1995; SCHIMLECK et al., 1999; SCHIMLECK et al., 2004; HEIN et al., 2009).

A variabilidade das características físico-químicas da madeira depende do gênero, espécie, procedência, condições edafoclimáticas, posição na árvore e, também, da idade. Em estudos sobre idade, Vital et al. (1984), Trugilho et al. (1996) e Silva et al. (2005) analisaram o efeito dessa variável nas características físico-químicas e anatômicas da madeira. A densidade tende a aumentar com a idade, como consequência do incremento da espessura da parede celular e da diminuição na largura das células. Em alguns estudos com árvores de Eucalyptus spp. de até 7 anos, o teor da lignina é propenso a diminuir com a idade, pois espécies mais jovens tendem a possuir maior proporção de madeira juvenil, mais rica em lignina que a madeira madura. A mesma tendência ocorre também com relação ao teor de extrativos, que tende a diminuir até certa idade, estabiliza e, mais tarde, tendem a aumentar com o avanço da idade (TRUGILHO et al., 1996; MORAES, 2008).

O objetivo deste estudo foi desenvolver modelos de calibração multivariada NIRS para amostras de Eucalyptus em idades de 3 a 7 anos, para densidade básica e teores de lignina e extrativos em acetona, bem como averiguar a capacidade preditiva de cada modelo, por meio de validação externa com amostras diferentes das utilizadas na calibração.

\section{MATERIAL E MÉTODOS}

\subsection{Material}

Foram utilizadas 136 árvores clonais de Eucalyptus spp., sendo 21 árvores com idade de 3 anos, 40 árvores com idades entre 4 e 6 anos e 75 árvores com 7 anos de idade, todas provenientes de um mesmo local de plantio do Estado de São Paulo.

Três árvores de cada clone, com altura e DAP (Diâmetro à Altura do Peito) representando a média do povoamento, foram abatidas e cortadas em toretes

Revista Árvore, Viçosa-MG, v.37, n.6, p.1165-1173, 2013

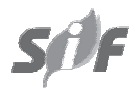


de $50 \mathrm{~cm}$ cada, na base, 25, 50, 75 e 100\% da altura comercial. Esses toretes foram processados em cavacos, por meio de um picador laboratorial e classificados em peneiras de 16 x 16 mm. Foi também realizada a classificação manual para retirada de nós da madeira e de cavacos mal dimensionados. Depois de selecionados, os cavacos foram secos ao ar e, em seguida, misturados em um homogeneizador rotatório. Depois de misturados, foram colocados em sacos de polietileno hermeticamente fechados, para conservação e uniformização do teor de umidade.

Partes das amostras de cavacos de cada clone foram selecionadas para determinação da densidade básica, segundo a norma da ABNT (NBR 11941). Outro grupo de amostras foi selecionado para ser transformado em serragens, com o uso de um moinho Willey. As serragens foram classificadas em peneiras de 40 e 60 mesh e usadas na determinação dos teores de lignina total (GOLDSCHMID, 1971; GOMIDE; DEMUNER, 1986) e extrativos (TAPPI T264 om-82).

\subsection{Preparo de amostras para leituras no espectrofotômetro NIR}

As serragens que passaram pela peneira de 40 mesh e ficaram retidas na peneira de 60 mesh foram novamente moídas em moinho tipo ciclone, para uniformização da granulometria, e, a seguir, acondicionadas em local com umidade e temperatura controladas ( $50 \%$ e $24^{\circ} \mathrm{C}$ ). Após $24 \mathrm{~h}$ de acondicionamento, a serragem foi compactada manualmente na unidade de leitura do espectrofotômetro e foram coletados os espectros NIR, tendo sido realizadas duas leituras de cada amostra. O espectrofotômetro utilizado foi o NirSystem 5000 da Foss, com o auxílio do software WinISI ${ }^{\circledR}$, que obtém espectros a cada $2 \mathrm{~nm}$ de comprimento de onda, no intervalo de 1.100 a $2.500 \mathrm{~nm}$, determinando a refletância para 700 valores de comprimento de onda. O espectrofotômetro realiza 32 varreduras por leitura e envia o valor médio de log (1/R) para o computador, gerando, assim, o espectro da amostra (NISGOSKI, 2005).

\subsection{Calibrações e validações NIRS}

Os dados espectrais foram exportados do Programa WinISI ${ }^{\circledR} 1.5$ para o The Unscrambler ${ }^{\circledR}$ 9.6 (CAMO S.A.), em que foram determinadas as médias das leituras dos espectros duplicados e realizadas as análises estatísticas. As calibrações foram desenvolvidas utilizando regressões de mínimos quadrados parciais (PLS), com validação cruzada completa e um máximo de 15 fatores.
O número de fatores adotado para cada modelo foi o valor que minimizou o erro-padrão médio (RMSECV) e maximizou o coeficiente de determinação da validação cruzada ( $\left.R_{c v}^{2}\right)$. Foram também testadas transformações matemáticas espectrais propostas por Savitzky e Golay (1964). Amostras anômalas, com alto "leverage” e alta variância residual, visivelmente diferentes do restante das amostras, foram detectadas em análises gráficas como outliers e excluídas dos modelos.

Foram desenvolvidos três tipos de calibrações NIRS separadas por idade das amostras: (i) idade de 7 anos; (ii) de 3 e 7 anos; e (iii) de 3 a 7 anos (Tabela 1). Os modelos gerados somente com amostras de 7 anos de idade foram utilizados para validar a densidade básica, teores de lignina e extrativos da madeira das amostras de 3 anos de idade e amostras com idades de 4 a 6 anos. Modelos NIRS gerados com amostras de 3 e 7 anos foram utilizados para validar amostras de 3, 7 e 4 a 6 anos de idade, e, por fim, os modelos desenvolvidos com amostras de todas as idades foram usados na predição de amostras de 3 a 7 anos.

Para a predição foram utilizados grupos de amostras que não fizeram parte das calibrações dos modelos NIRS, sendo conhecidos como validação externa (BURNS; CIURCZAK, 2008). As amostras da validação externa tiveram suas características determinadas em laboratório e comparadas com as predições via NIRS.

\subsection{Seleção das calibrações}

Análises estatísticas foram utilizadas para seleção das calibrações NIRS. Utilizaram-se os coeficientes de determinação da calibração $\left(R_{c}^{2}\right)$ e da validação cruzada ( $R_{c v}^{2}$ ), os erros-padrão da calibração (SEC) e da calibração cruzada (SECV) e a relação de desempenho do desvio (RPD). Segundo Williams e Sobering (1993), o RPD é uma técnica utilizada para verificar a precisão de calibração de um modelo, sendo calculado como a razão entre o desvio-padrão dos valores de referência

Tabela 1 - Calibrações NIRS.

Table 1 - NIRS calibrations.

\begin{tabular}{|c|c|}
\hline Calibrações & Grupo de Validação \\
\hline Amostras de 7 anos & $\begin{array}{c}\text { Amostras de } 3 \text { anos } \\
\text { Amostras de } 4 \text { a } 6 \text { anos }\end{array}$ \\
\hline Amostras de 3 e 7 anos & $\begin{array}{l}\text { Amostras de } 3 \text { e } 7 \text { anos } \\
\text { Amostras de } 4 \text { a } 6 \text { anos }\end{array}$ \\
\hline Amostras de 3 a 7 anos & Amostras de 3 a 7 anos \\
\hline
\end{tabular}


e o erro padrão da validação cruzada (SECV) ou erro padrão da validação externa (SEP). Assim, quanto maior o valor do RPD, melhor o ajuste do modelo (FUJIMOTO et al., 2008).

Análises estatísticas similares foram usadas para validação externa, utilizando-se o coeficiente de determinação da predição $\left(R_{p}^{2}\right)$ e o $R P D_{p}$, que é a razão entre o desvio-padrão dos valores de referência e o erro-padrão da predição.

\section{RESULTADOS}

Para melhor visualização dos espectros das 136 amostras de madeira, na Figura 1 pode ser observado o gráfico de "scores” da análise de componentes principais. Como pode ser visto, houve separação dos grupos, tendo as amostras de 4 a 6 anos sido localizadas em nível mais alto do eixo PC2, as amostras de 7 anos num nível mais baixo e entre esses dois grupos ficaram as amostras com idades de 3 anos.

\subsection{Calibrações NIRS}

Na Tabela 2 estão apresentados os resultados das análises estatísticas descritivas das 136 amostras.

Como pode ser observado na Tabela 2, em média as madeiras de 3 anos de idade apresentaram, quando comparadas com as de 4 a 6 anos e de 7 anos, densidade básica mais baixa, maior teor de lignina e teor de extrativos intermediários.
Os parâmetros estatísticos dos modelos NIRS estabelecidos neste estudo nas diferentes idades das madeiras são apresentados na Tabela 3 .

Os resultados das calibrações dos três grupos de idades mostraram que os modelos de densidade básica alcançaram elevado $R^{2}$. A qualidade dos modelos é demonstrada pelos altos $R_{c}^{2}$, pelos valores de $R P D$ (superior a 2), pelos coeficientes de determinação da $R_{c v}^{2}$, pelos baixos erros de $S E C$ e erros de $S E C V$, com valores mínimos de 10 e máximos de $18 \mathrm{~kg} / \mathrm{m}^{3}$, respectivamente.

Os modelos do teor de lignina apresentaram parâmetros mais baixos, em relação ao $R_{c v}^{2}(0,71$ a 0,55$)$ e ao $R P D(1,97$ a 1,45$)$. Nesses modelos, o $S E C$ foi inferior a $1,0 \%$ e o SECV apresentou erro máximo de $1,1 \%$.

Em relação aos teores de extrativos das madeiras, os $R_{c}^{2}$ foram os melhores entre os modelos dos outros dois parâmetros analisados e variaram de um máximo de 0,98 nas idades de 3 e 7 anos, a um mínimo de 0,92 na idade de 7 anos. $\mathrm{O} R_{c v}^{2}$ alcançou maior valor no modelo com amostras de 3 e 7 anos de idade e menor naquele com idade de 3 a 7 anos.

\subsection{Predições NIRS}

Os modelos desenvolvidos foram utilizados para predições, bem como testados os desempenhos preditivos das calibrações de amostras de diferentes idades, cujos resultados são mostrados nas Figuras 2.

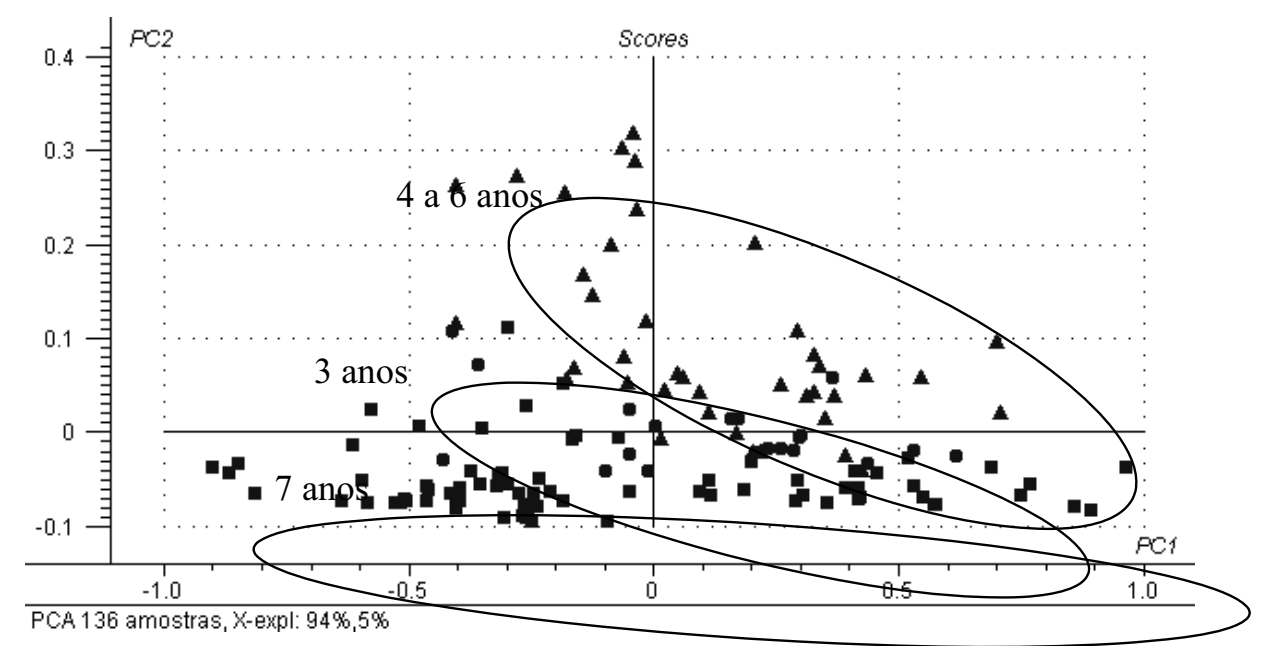

Figura 1 - Análise de componentes principais das amostras de 3(•), 7(•) e 4-6(《) anos de idade.

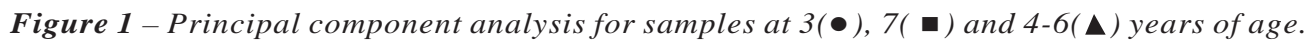

Revista Árvore, Viçosa-MG, v.37, n.6, p.1165-1173, 2013 
Tabela 2 - Análises estatísticas abrangendo valores de máximo, mínimo e média, desvio padrão (DP) e coeficiente de variação (CV) dos parâmetros estudados.

Table 2 - Summary statistics of maximum, minimum, average, standard deviation (SD) and coefficients of variation (CV) of the parameters studied.

\begin{tabular}{|c|c|c|c|c|c|c|c|}
\hline Amostras (idade) & Número & Parâmetros & Máximo & Mínimo & Média & $\mathrm{DP}$ & CV (\%) \\
\hline \multirow[t]{3}{*}{3 anos } & 21 & Densidade básica $\left(\mathrm{kg} / \mathrm{m}^{3}\right)$ & 483 & 367 & 432 & 32 & 7 \\
\hline & & Lignina total (\%) & 30,2 & 26,2 & 28,2 & 1,1 & 3,9 \\
\hline & & Extrativos (\%) & 3,4 & 1,4 & 2,7 & 0,6 & 23,7 \\
\hline \multirow[t]{3}{*}{4 a 6 anos } & 40 & Densidade básica $\left(\mathrm{kg} / \mathrm{m}^{3}\right)$ & 519 & 353 & 435 & 42 & 10 \\
\hline & & Lignina total (\%) & 29,6 & 25,2 & 27,9 & 1,0 & 3,7 \\
\hline & & Extrativos (\%) & 2,4 & 0,9 & 1,5 & 0,4 & 22,8 \\
\hline \multirow[t]{3}{*}{7 anos } & 75 & Densidade básica $\left(\mathrm{kg} / \mathrm{m}^{3}\right)$ & 555 & 429 & 493 & 26 & 5 \\
\hline & & Lignina total (\%) & 30,9 & 23,3 & 27,3 & 1,8 & 6,5 \\
\hline & & Extrativos (\%) & 7,3 & 1,2 & 3,1 & 1,3 & 41,2 \\
\hline \multirow[t]{3}{*}{ Total } & 136 & Densidade básica $\left(\mathrm{kg} / \mathrm{m}^{3}\right)$ & 555 & 353 & 466 & 44 & 9 \\
\hline & & Lignina total (\%) & 30,9 & 23,3 & 27,6 & 1,5 & 5,5 \\
\hline & & Extrativos (\%) & 7,3 & 0,9 & 2,6 & 1,2 & 46,7 \\
\hline
\end{tabular}

Tabela 3 - Parâmetros estatísticos dos modelos NIRS desenvolvidos para as diferentes idades. Table 3 -Summary statistics for the NIRS models developed for different ages.

\begin{tabular}{|c|c|c|c|c|c|c|c|c|c|}
\hline Idade & Características químicas & Número de amostras & "Outlier” & $\mathrm{VL}$ & $\mathrm{R}_{c}^{2}$ & $\mathrm{R}_{\mathrm{cV}}^{2}$ & SEC & SECV & RPDc \\
\hline \multirow[t]{3}{*}{7 anos } & Densidade básica $\left(\mathrm{kg} / \mathrm{m}^{3}\right)$ & 72 & 3 & 7 & 0,84 & 0,76 & 10 & 13 & 2,04 \\
\hline & Lignina total (\%) & 72 & 3 & 5 & 0,80 & 0,71 & 0,8 & 0,9 & 1,97 \\
\hline & Extrativos (\%) & 75 & - & 6 & 0,92 & 0,90 & 0,4 & 0,4 & 3,10 \\
\hline \multirow[t]{3}{*}{3 e 7 anos } & Densidade básica $\left(\mathrm{kg} / \mathrm{m}^{3}\right)$ & 68 & 3 & 7 & 0,87 & 0,80 & 13 & 16 & 2,24 \\
\hline & Lignina total (\%) & 67 & 4 & 8 & 0,88 & 0,56 & 0,6 & 1,1 & 1,48 \\
\hline & Extrativos (\%) & 71 & - & 8 & 0,98 & 0,92 & 0,2 & 0,3 & 3,95 \\
\hline \multirow[t]{3}{*}{3 a 7 anos } & Densidade básica $\left(\mathrm{kg} / \mathrm{m}^{3}\right)$ & 104 & 2 & 7 & 0,89 & 0,83 & 14 & 18 & 2,41 \\
\hline & Lignina total (\%) & 100 & 6 & 7 & 0,72 & 0,55 & 0,8 & 1,0 & 1,45 \\
\hline & Extrativos (\%) & 104 & 2 & 9 & 0,95 & 0,86 & 0,3 & 0,4 & 2,97 \\
\hline
\end{tabular}

O modelo desenvolvido para a idade de 7 anos foi testado para predizer as amostras de 3 anos e também o grupo de amostras de 4 a 6 anos. Na Figura 2A são mostrados os coeficientes de determinação da predição ( $R_{p}^{2}$ ) desses dois grupos e na Figura 2B, as comparações das relações de desempenho do desvio $\left(R P D_{P}\right)$ de cada grupo de predição.

O modelo desenvolvido com amostras de 3 e 7 anos de idade foi utilizado para predizer amostras com idades de 3 e 7 anos e também amostras com idades de 4 a 6 anos, como observado na Figura 2CD, em que são mostradas comparações de $R_{p}^{2}$ e $R P D_{P}$. Todas as três características analisadas nas madeiras de 3 e 7 anos apresentaram valores de $R^{2} \mathrm{p}$ acima de 0,60 , sendo 0,82 para densidade básica e 0,89 para o teor de extrativos. Para o grupo de madeiras com idades de 4 a 6 anos, os valores de $R_{p}^{2}$ foram baixos, exceto a densidade básica, que demonstrou elevado valor de $R_{p}^{2}(0,82)$.

Nas Figuras 2EF são mostrados os $R_{p}^{2}$ e os $R P D_{P}$, respectivamente, para predição das amostras de 3 a 7 anos de idade, utilizando o modelo desenvolvido, especificamente, com amostras de madeiras com as mesmas idades. O $R_{p}^{2}$ dos extrativos foi muito preciso, com valor de 0,90 , a densidade básica alcançou valor de 0,83 e a lignina atingiu o menor valor, 0,48 . $O R P D_{P}$ apresentou a mesma tendência do $R_{p}^{2}$, mas o modelo usado para a predição de lignina com idade de 3 a 7 anos não foi satisfatório, apresentando um $R P D_{P}$ igual a 1,00.

\section{DISCUSSÃO}

Segundo Trugilho et al. (1996), após os 3 anos de idade existe tendência de estabilização de algumas

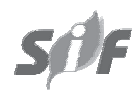

Revista Árvore, Viçosa-MG, v.37, n.6, p.1165-1173, 2013 

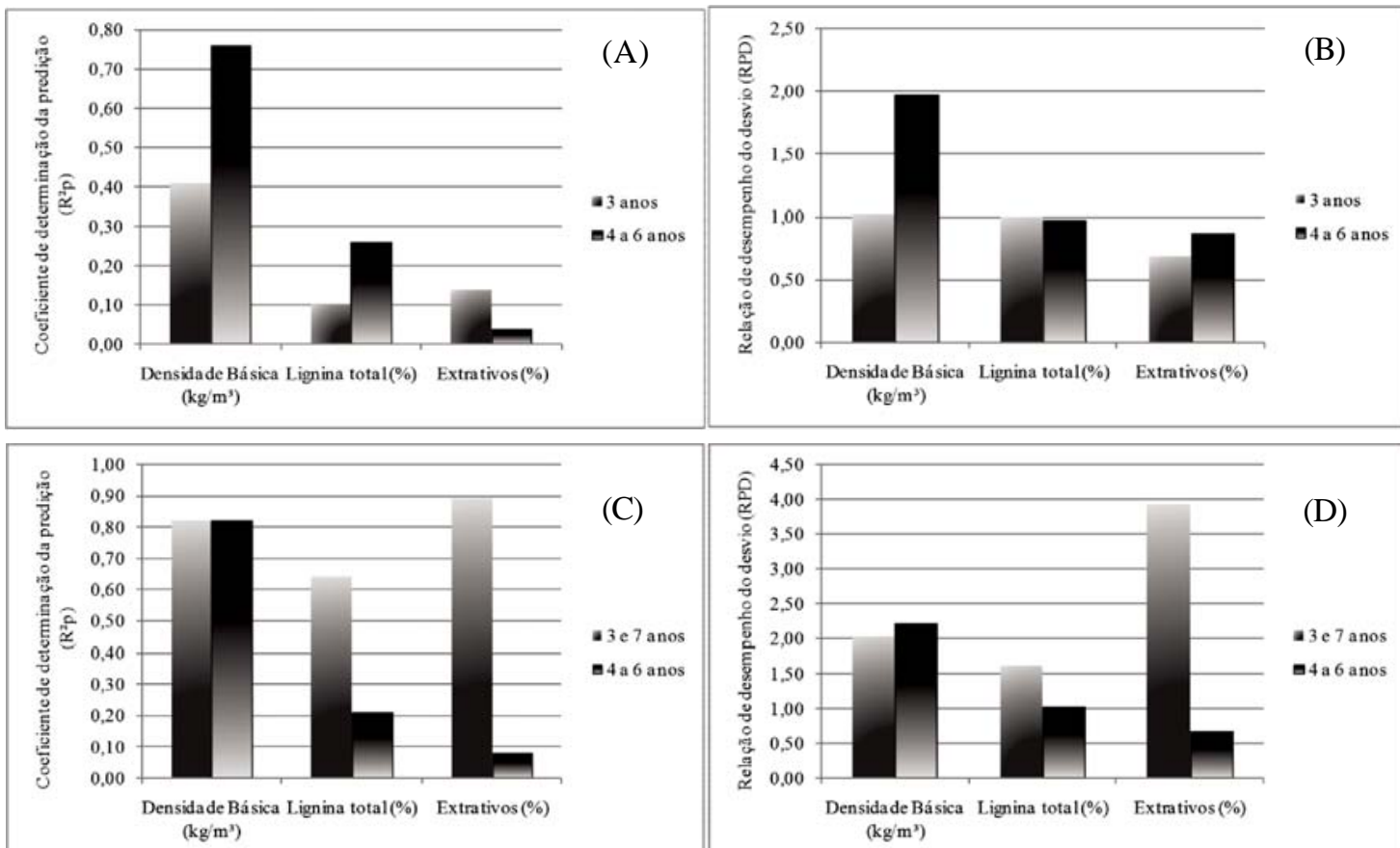

(C)
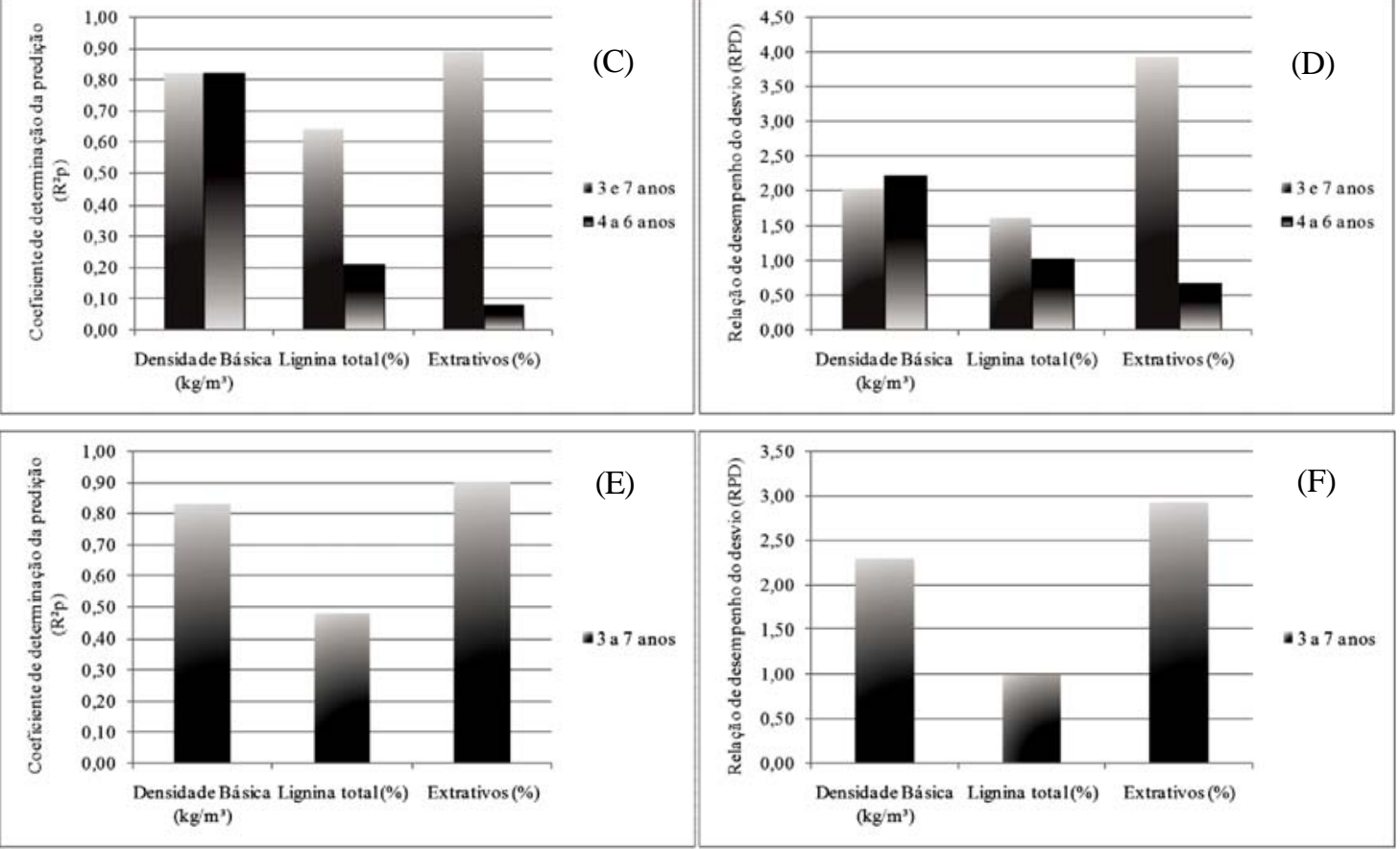

Figura 2 - Coeficiente de determinação da predição $\left(\mathrm{R}^{2} \mathrm{p}\right)$ e relação de desempenho de desvio (RPDp) dos modelos desenvolvidos com amostras de: (A e B) 7 anos de idade (C e D), 3 e 7 anos de idade e (E e F) 3 a 7 anos de idade.

Figure 2 - Coefficient of prediction determination $\left(R^{2} p\right)$ and ratio of performance deviation for prediction $(R P D p)$ for the models developed with samples: ( $A$ and $B) 7$ years old ( $C$ and $D), 3$ and 7 years old and $(E$ and $F)$ 3-7 years old.

características físico-químicas da madeira de algumas espécies de Eucalyptus, como a densidade básica e os teores de lignina e de extrativos, o que sugere o início da formação da madeira adulta. Estudos têm demonstrado que o teor de extrativos tende a diminuir até os 4 anos de idade (TRUGILHO et al., 1996) e a aumentar depois de cinco anos ( SILVA et al., 2005; MORAES, 2008), devido ao processo de cernificação da madeira.

Os resultados dos parâmetros estatísticos dos modelos da densidade básica possibilitarão, certamente, adequada predição para estudos de melhoramento florestal. Schimleck et al. (2006) encontraram valores de $R_{c}^{2}$ para densidade básica variando de 0,76 a 0,64 , enquanto Tyson et al. (2009), valores de $R_{c}^{2}$ iguais a 0,75 e $R P D_{C}$ de 1,71 , inferiores aos encontrados neste trabalho, bem como erros mais elevados com SEC de $27 \mathrm{~kg} / \mathrm{m}^{3}$ e $S E C V$ de $32 \mathrm{~kg} / \mathrm{m}^{3}$. Outros resultados de densidade básica também foram determinados por Cogdill et al. (2004), com $R_{c}^{2}$ de 0,81 e erros de $S E C$ e $S E C V$ de 35 e $39 \mathrm{~kg} / \mathrm{m}^{3}$, respectivamente. 
Em relação ao teor de lignina total, Tyson et al. (2009), em seus estudos com 140 árvores de Eucalyptus, encontraram valores intermediários aos deste estudo, com $R_{c}^{2}$ de 0,76 e erro $S E C$ e $S E C V$ de 0,7 e $0,8 \%$, respectivamente, sendo considerados pelos referidos autores como resultados satisfatórios. Tal fato confirma ainda a qualidade dos parâmetros dos modelos para lignina, que neste estudo foram as correlações encontradas no trabalho de Schimleck et al. (2006), com $R_{c}^{2}$ de 0,79, erros SEC e SECV de 0,8 e 0,9\% e RPDp de 1,90.

O desempenho dos modelos desenvolvidos com amostras de 7 anos de idade para predição de amostras com 3 anos não apresentou precisão aceitável, uma vez que as predições exibiram $R_{p}^{2}$ de 0,41 para a densidade básica, 0,10 para lignina e 0,14 para os extrativos. Nas madeiras com idades de 4 a 6 anos, o modelo também não mostrou alto desempenho de predição para as características de lignina e extrativos, tendo apresentado $R_{p}^{2}$ abaixo de 0,30 , e somente para densidade básica o modelo alcançou $R_{p}^{2}$ de 0,76 , sendo este considerável. O RPDp para densidade básica teve o maior valor, próximo a 2, indicando que o modelo estabelecido para essa propriedade seria suficientemente preciso na seleção de amostras, desde que as madeiras tenham idades de 4 a 6 anos. De acordo com alguns autores (TRUGILHO et al., 1996; ALENCAR et al., 2002; MORAES, 2008), ocorre nas madeiras de Eucalyptus tendência de redução da taxa de incremento da densidade básica com o aumento da idade, fenômeno que pode ter contribuído para a melhor predição das amostras com idades de 4 a 6 anos. As amostras com idades de 3 anos não foram bem preditas com o modelo de 7 anos para nenhuma das características analisadas, uma vez que todos os $R P D_{P}$ foram iguais ou inferiores a 1 .

Para predição das amostras de madeira com idades de 3 e 7 anos, foi alcançado $R P D_{P}$ de 3,93 para o teor de extrativos, considerado muito satisfatório para predição em melhoramento florestal, mas a lignina e a densidade básica apresentaram valores bem inferiores (1,60 e 2,03, respectivamente). A utilização do modelo com amostras de 3 e 7 anos para predizer amostras com idades de 4 a 6 anos não demonstrou bom desempenho, tendo apresentado $R P D_{P}$ para os teores de lignina e extrativos de 1,03 e 0,67, respectivamente; no caso da densidade básica, esta teve o melhor valor de $R P D_{P}(2,22)$. O fato dos resultados para o teor de extrativos não terem apresentado precisão suficiente ao utilizar um modelo de 3 e 7 anos para predizer amostras de 4 a 6 anos pode ser explicado pela queda no incremento do teor de extrativos até cerca de 5 anos de idade e, após essa idade, pela ocorrência de elevação do teor de extrativos novamente (MORAES, 2008).

\section{CONCLUSÃO}

Pela análise de componentes principais, as madeiras analisadas apresentaram diferenças espectrais entre as idades.

Os resultados deste estudo indicaram boas correlações dos modelos desenvolvidos para cada grupo de amostras, separadas por idade, exceto no teor de lignina. A escolha do modelo para predição de amostras deve ser de acordo com as características das amostras a serem preditas, ou seja, amostras com idades diferentes das utilizadas no desenvolvimento do modelo não proporcionarão boas predições. Neste estudo, entretanto, apenas para densidade básica os modelos desenvolvidos para a idade de 7 anos e modelos desenvolvidos para idades de 3 e 7 anos predisseram com precisão suficiente as amostras com idades de 4 a 6 anos.

A incorporação de amostras de várias idades (3 a 7 anos), representativas de cada grupo, permitiu o desenvolvimento de modelos capazes de predizer amostras de diferentes idades com melhorias no desempenho das predições.

\section{REFERÊNCIAS}

\section{ASSOCIAÇÃO BRASILEIRA DE NORMAS} TÉCNICAS - ABNT. Norma NBR 11941: Madeira: determinação da densidade básica. Rio de Janeiro: 2003. 6p.

\section{ALENCAR, G. S. B. Estudo da qualidade da madeira para produção de polpa celulósica relacionada à precocidade na seleção de um Híbrido Eucalyptus grandis x Eucalyptus urophylla. 2002. $73 f$. Dissertação (Mestrado em Ciências Florestais) - Escola Superior de Agricultura "Luiz de Queiroz”, Universidade de São Paulo, Piracicaba, 2002.}

ALVES, A. et al. Calibration of NIR to assess lignin composition (H/G ratio) in maritime pine wood using analytical pyrolysis as the reference method. Holzforschung, v.60, n.1, p.29-31, 2006.

Revista Árvore, Viçosa-MG, v.37, n.6, p.1165-1173, 2013 
BIRKETT, M. D.; GAMBINO, M. J. T. Estimation of pulp kappa number with near infrared spectroscopy. Tappi Journal, v.72, n.9, p.193197, 1998.

BOERIU, C. G. et al. Characterisation of structuredependent functional properties of lignin with infrared spectroscopy. Industrial Crops and Products, v.20, n.2, p.205-218, 2004.

BOKOBZA, L. Near infrared spectroscopy.

Journal of Near Infrared

Spectroscopy, v.6, n.1, p.3-17, 1998.

ASSOCIAÇÃO BRASILEIRA DE CELULOSE E PAPEL - BRACELPA. Panorama do setor. 2012. Disponível em: <http://www.bracelpa.org.br/bra2/ $>$ Acesso em 18 de mar. 2013.

BRINKMANN, K.; BLASCHKE, L.; POLLE, A. Comparison of different methods for lignin determination as a basis for calibration of nearinfrared reflectance spectroscopy and implications of lignoproteins. Journal of Chemical Ecology, v.28, n.12, p.2483-2501, 2002.

BURNS, D. A.; CIURCZAK, E. W. Handbook of near-infrared analysis. 3.ed. Bоса Raton: CRC, 2008. 808p.

COGDILL, R. P. et al. Estimation of the physical wood properties of Pinus taeda L. radial strips using least squares support vector machines.

Journal of Near Infrared

Spectroscopy, v.12, n.4, p.263-269, 2004.

EASTY, D. B. et al. Near-infrared spectroscopy for the analysis of wood pulp: quantifying hardwoodsoftwood mixtures and estimating lignin content. Tappi Journal, v.73, n.10, p.257-261, 1990.

FUJIMOTO, T. et al. Application of near infrared spectroscopy for estimating wood mechanical properties of small clear and full length lumber specimens. Journal of Near Infrared Spectroscopy, v.16, n.6, p.529-537, 2008.

GIERLINGER, N. et al. Rapid determination of heartwood extractives in Larix sp. by means of Fourier transform near infrared spectroscopy.

Journal of Near Infrared

Spectroscopy, v.10, n.3, p.203-214, 2002.
GOLDSCHIMID, O. Ultravioleta spectra. In: SARKANEM, K.; LUDWING, C. H. Lignins: ocurrence, formation, structure and reactions. New York: John Wiley \& Sons, 1971. p.241-298.

GOMIDE, J. L.; DEMUNER, B. J. Determinação do teor de lignina na madeira: método Klason modificado. O Papel, v.47, n.8, p.36-38, 1986.

HEIN, P. R. G. et al. Near infrared spectroscopy for estimating wood basic density in Eucalyptus urophylla and Eucalyptus grandis. Revista Cerne, v.15, n.2, p.133-141, 2009.

HOFFMEYER, P.; PEDERSEN, J. G. Evaluation of density and strength of Norway spruce by near infrared reflectance spectroscopy. Holz als Roh- und Werkstoff, v.53, n.3, p.165-170, 1995.

MORAES, P. H. D. Efeito da idade da madeira de eucalipto na sua química e polpabilidade, e branqueabilidade e propriedades físicas da polpa. 2008. 79f. Dissertação (Mestrado em Agroquímica) Universidade Federal de Viçosa, Viçosa, MG, 2008.

NISGOSKI, S. Espectroscopia no infravermelho próximo no estudo de características da madeira e papel de Pinus taeda L. 2005. 173f. Tese (Doutorado em Engenharia Florestal) - Universidade Federal do Paraná, Curitiba, 2005.

SAVITZKY, A.; GOLAY, M. J. E. Smoothing and differentiation of data by simplified least squares procedures. Analytical Chemistry, v.36, n.8, p.1627-1639, 1964.

SCHIMLECK, L. R. et al. Estimation of whole-tree wood quality traits using near infrared spectra collected from increment cores. Appita Journal, v.59, n.3, p.231-236, 2006.

SCHIMLECK, L. R. et al. Development of wood property calibrations using near infrared spectra having different spectral resolutions. Journal of Near Infrared Spectroscopy, v.12, n.1, p.55-61, 2004.

SCHIMLECK, L. R. et al. Estimation of basic density of Eucalyptus globulus using nearinfrared spectroscopy. Canadian Journal of Forest Research, v.29, n.2, p.194-201, 1999. 
SILVA, J. C. et al. Influência da idade e da posição ao longo do tronco na composição química da madeira de Eucalyptus grandis Hill ex. Maiden. Revista Árvore, v.29, n.3, p.455-460, 2005.

SILVA JÚNIOR, F. G.; BRAGA, E. P.

Potencialidade da seleção precoce de Eucalyptus urophylla em função da qualidade da madeira destinada à produção de celulose. São Paulo:

Associação Brasileira Técnica de Celulose e Papel - ABTCP, 1997.

TECHNICAL ASSOCIATION OF THE PULP AND PAPER INDUSTRY - TAPPI. Standard

Methods of Technical Association of the Pulp and Paper Industry. Atlanta: 2002.

TRUGILHO, P. F.; LIMA, J. T.; MENDES, L. M. Influência da idade nas características físicoquímicas e anatômicas da madeira de Eucalyptus saligna. Revista Cerne, v.2, n.1, p.94-111, 1996.
TYSON, J. A. et al. Adjusting near infrared wood property calibrations for central Brazil to predict the wood properties of samples from southern Brazil. Journal Appita, v.62, n.1, p.46-51, 2009.

VITAL, B. R.; PEREIRA, A. R.; DELLA LUCIA, R. $M$. Efeito da idade da árvore na densidade da madeira de Eucalyptus grandis cultivado na região de serrado de Minas Gerais. Brasília. Brasília: IBDF, 1984. p.41-52.

WILLIAMS, P. C.; SOBERING, D. C. Comparison of commercial near infrared transmittance and reflectance instruments for analysis of whole grains and seeds. Journal of Near Infrared Spectroscopy, v.1, n.1, p.25-33, 1993.

WRIGHT, J.; BIRKETT, M.; GAMBINO, M. Prediction of pulp yield and cellulose content from wood using near infrared reflectance spectroscopy. Tappi Journal, v.73, n.8, p.164166, 1990. 
